\title{
Effect of Anesthetic Drugs on Vestibular Evoked Myogenic Potential Recording
}

\author{
Gaetano Motta ${ }^{1}$, Giuliano Sequino ${ }^{2}$, Antonella Menafro ${ }^{3}$, Giancarlo Vesce ${ }^{3}$, Giuseppina Marcuccio ${ }^{*}, 1$, \\ Antonello Brancaccio ${ }^{1}$, Arturo Lecce ${ }^{1}$ and Domenico Testa ${ }^{1}$ \\ ${ }^{1}$ Institute of Otorhinolaryngology - Second University of Naples, Italy \\ ${ }^{2}$ Institute of Otorhinolaryngology - University of Naples "Federico II", Italy \\ ${ }^{3}$ Department of Veterinary Clinical Sciences - University of Naples "Federico II", Italy
}

\begin{abstract}
The human saccule has preserved the ancestral ability to respond to high-intensity acoustic stimulations, generating a contraction reflex of the cervical tract muscles. Recording the muscular bioelectric potentials following saccular stimulation allows the evidence of the vestibular-evoked myogenic potentials (VEMPs).

The aim of this study was to record VEMPs in alert and anesthetized guinea pigs to evaluate the possible different impacts of some anesthetic drugs on VEMP recording.

Sixteen guinea pigs, divided into four groups, were employed for the study. Each group underwent general anesthesia induced using different pharmacological regimens; auditory and vestibular functions were examined with Auditory Brain Response (ABR) and VEMP methods.

The analysis of the results showed that the VEMPs recording performance was strictly related to the alert status of the guinea pigs: VEMPs were not recordable during anesthesia while reappeared at awakening, with the same temporal and morphological characteristics of pre-anesthesia.

ABR was instead normally present during anesthesia without showing any differences with all the various anesthetic drugs employed.

These results prompted us to conclude that anesthetic drugs cause the disappearance of saccular reflex in guinea pigs, not minding the specific type of drugs employed. This evidence, in agreement with the data available in literature for humans, induces and encourages future studies about sacculocollic reflex and its possible application in clinical practice.
\end{abstract}

Keywords: VEMPs, ABR, anesthetic drugs.

\section{INTRODUCTION}

Saccular receptors undergoing adequate stimuli trigger a sacculocollic reflex. The afferent pathway starts from saccular receptors, and, through the inferior vestibular nerve, reaches the Deiters' lateral vestibular nucleus. The efferent pathway goes from Deiters' nucleus to spinal $\alpha$-motoneurons of cervical tracts $\mathrm{C} 2-\mathrm{C} 5$; the motor fibers reach the ipsilateral sternocleidomastoid muscle through the external branch of the accessory spinal nerve. The saccular reflex can be recorded as a variation of the bioelectric potential and indicates the integrity of the inferior vestibular nerve [1-3]. The main proof of the vestibular origin of this potential is based on its presence also in patients with severe sensorineural hearing loss and normal vestibular receptors [4].

Animal models have been used to investigate the origin of the saccular reflex in physiological, pathological and pharmacologically-induced conditions [5]. Matsutzaki and

*Address correspondence to this author at the Institute of Otorhinolaryngology, Second University of Naples, via San Marco, 5 81010 Castel Campagnano, Caserta, Italy; Tel/Fax: +393471393603;

E-mail: giuseppina_marcuccio@hotmail.it
Murofushi [6-8, 14, 15] recorded click-evoked myogenic potentials in the neck of anesthetized guinea pigs, interpreting them as a non-specific vestibular response.

In humans VEMPs have been recorded in anterior and posterior labyrinth pathologies [9], vestibular neuritis [10], benign paroxysmal positioning vertigo [11], hydropic disorders [12] and herpes zoster oticus [13]. However, up to now, the importance of the alert status on VEMP recording has been poorly studied.

The aim of this study was to record VEMPs in alert and anesthetized guinea pigs employing surface electrodes, to evaluate the following parameters: the presence of the sacculocollic reflex in relation to the alert status; the possible differences due to the various anesthetic drugs in the VEMP recordings; the relationship between VEMPs and the auditory function.

\section{MATERIALS AND METHODS}

The study was conducted jointly at the Department of Otorhinolaryngology of the Second University of Naples, the Department of Otorhinolaryngology of the University of Naples "Federico II", and the Biopharmacologic Unit of the Cardarelli Hospital, Naples, Italy. The study was carried out 
in conformity with the italian law (116/92) and approved by the Ethics Committee for Animal Experimentation (CESA) of the Cardarelli Hospital.

Sixteen female guinea pigs (weight $250-400$ gr) were selected and randomly assigned to one of the four groups created, each one made up of four units.

Each animal underwent ABR examination to evaluate the auditory threshold and functionality of the auditory system and VEMPs recording before, during and after anesthesia induction.

After an acclimatizing period, the animals were kept without food for six hours and without water for one hour before anesthesia.

Pre-anesthetic check-up included the evaluation of the health state; the measurement of respiratory and heart rate and temperature recording.

Each group underwent a specific anesthesiologic protocol:

- Group 1: $0.5 \mathrm{ml} / \mathrm{kg}$ of a mixture of acepromazine $(1.42 \mathrm{mg} / \mathrm{ml})$, xylazine $(8.57 \mathrm{mg} / \mathrm{ml})$ and ketamine ( $42.85 \mathrm{mg} / \mathrm{ml})$, intramuscular injection (i.m.);

- Group 2: Medetomidine $(0.5 \mathrm{mg} / \mathrm{kg})$ and ketamine (40 mg/kg), intra peritoneum (i.p.);

- Group 3: $80 \mathrm{mg} / \mathrm{kg}$ of a mixture of tiletaminezolazepam (Zoletil 100®), i.m.;

- Group 4: Diazepam (5mg/kg i.p.) followed by fentanyl $(0.3 \mathrm{mg} / \mathrm{kg}$ i.m. $)$ after ten minutes;

The time interval between the administration of the anesthetic drugs and the loss of consciousness was recorded as the 'induction time'. The 'effect length' was considered as the time interval between the loss of consciousness and the reappearance of spontaneous movements. The presence of palpebral and flexor reflexes (the latter consisting of manual compression of the metatarsus) and the heart and respiratory rate were recorded every 10 minutes.

The level of analgesia was evaluated observing the response to uniform painful stimuli, applied to the skin through $2-12 \mathrm{~mA}$ discharges (1ms) produced with an electric stimulator. The 'awakening time' was calculated as the lapse between the reappearance of spontaneous movements and the recovery from sternal decubitus. In some cases antagonistic drugs were administered, to evaluate the effect on timing and quality of awakening.

\section{ABR Test}

The recording of the evoked auditory potentials at the encephalic trunk was performed using a Medelec Amplaid MK 22 (MI) system (via Ripamonti, 133 Milano, Italy). Electrodes were set at the vertex (+); at ipsilateral mastoid (-) and on the median part of the muzzle (earth).

Alternated clicks were employed of $0.1 \mathrm{~ms}$, sweep rate $21 / \mathrm{s}$, decreasing intensity in $10 \mathrm{~dB}$ steps, starting from 110 $\mathrm{dB}$ SPL, presented ipsilaterally through TDh 4 headphones (Telephonics), bandpass filtered 100-3000 Hz, 2048 stimuli, analysis time $12 \mathrm{msec}$.
The amplitude and the absolute and inter-peak latencies were evaluated and recorded.

\section{VEMP Test}

Anesthetized guinea pigs were set, on proper support, in prone position, with the head raised for pre-activation of both sternocleidomastoid muscles. The recording was performed using Medelec Amplaid MK 22 (MI) system (via Ripamonti, 133 Milano, Italy).

The positive electrode was set either on the semispinal muscle (third cervical vertebra) and on both long extensor neck muscles, while the earth electrode was set on the median line of the occipital area. The signal was evoked by Logon, with $130 \mathrm{~dB}$ SPL intensity, presented ipsilaterally through TDh 4 headphones (Telephonics), sweep rate 5/s, bandpass filtered $30-3000 \mathrm{~Hz}, 200$ stimuli, analysis time 100 msec.

Each animal underwent a series of test-retest recordings of VEMPs at different levels of anesthetic awakening.

\section{Statistical Analysis}

The results were evaluated using there with Student's ttest. Correlations were analyzed both for the VEMPs responses and the ABR responses. The relevant level set in our study was $\mathrm{p}<0,05$.

\section{RESULTS}

\section{Anesthesia}

Group 1: The acepromazine, xylazine and ketamine mixture abolished the straightening reflex in 2 minutes; loss of consciousness was observed as early as the fifth minute, together with the disappearance of palpebral and flexor reflexes. Heart rate was $242 \pm 54$ b.p.m. and respiratory rate $55 \pm 7$ acts per minute. The analgesia level was acceptable (absence of responses until $10 \mathrm{~mA}$ ) and the muscular tone was almost abolished. Awakening started after about two hours and an half. In two animals in this group, after 60 minutes of anesthesia, atapimazole was administered $(0.5 \mathrm{mg}$ i.m.) to antagonize part of the effects of xylazine: awakening started after 5 minutes.

Group 2: The straightening reflex disappeared after 2 minutes, whereas palpebral and flexor reflexes persisted until the tenth minute. Anesthesia was characterized by good myoresolution and analgesia (absence of responses to stimuli after the twelfth minute). Heart rate was $216 \pm 47$ b.p.m. and respiratory rate $72 \pm 18$ acts per minute. Spontaneous awakening started after about one hour and took $45 \mathrm{~min}$. In two animals, the effect of medetomidine was antagonized by $2 \mathrm{mg} / \mathrm{kg}$ i.m. of atapimazole, which induced awakening after 30 minutes.

Group 3: The mixture of tiletamine-zolazepam (Zoletil ${ }^{\circledR}$ ) induced a weak dissociation with rigidity and low analgesia which lasted for two hours. The straightening reflex disappeared after 2 minutes, while palpebral and flexor reflexes persisted. The heart rate was $250 \pm 42$ b.p.m. and respiratory rate $72 \pm 14$ acts per minute. Awakening took one hour. The effects of Zolazepam were antagonized in three subjects with $0.5 \mathrm{mg} / \mathrm{kg}$ i.p. flumazenil one hour after induction and awakening was observed after 30 minutes. 
Group 4: Low sedation after administration of diazepam was observed. Fentanyl abolished straightening and palpebral reflexes after 5 minutes and flexor reflexes after 10 minutes. Anesthesia was accompanied by hispid hair. Heart rate was $220 \pm 45$ b.p.m. and respiratory rate $70 \pm 23$ acts per minute. Deep analgesia was observed but muscular tone was not completely abolished. Forty minutes after induction, muscular tremors followed by a slow recovery of spontaneous movements were observed. Flumazenil (0.6 $\mathrm{mg} / \mathrm{kg}$ i.p.) and naxolone ( $0.8 \mathrm{mg} / \mathrm{kg}$ i.m.) were administered in two animals, with awakening after five minutes.

\section{ABR}

In all cases $\mathrm{ABR}$ were normal before, during and after anesthesia (Fig. 1).

Tables 1 and 3 shows the absolute and inter-peak latencies average in the anesthetized guinea-pigs, divided in four groups in relation to the anesthesiologic protocol employed.

\section{VEMPs}

During anesthesia, intentionally induced without administering any curare drug, it was not possible to record sacculocollic reflexes, regardless of the anesthetics employed. Tables $\mathbf{2}$ and $\mathbf{4}$ shows the responses in the guinea pigs before and after anesthesia. In this table we can notice the absence of differences of VEMPs recordings before and after anesthesia; while, during anesthesia, VEMPs were not present. Figs. $(2,3)$ present the VEMPs results in the guinea pigs before, during and at complete awakening.

\section{DISCUSSION}

Several studies have shown that a high intensity sound or an intense vibration cause a vestibular reflex or an illusion of movement, due to the ancestral attidude of the saccule of playing the role of an acoustic receptor and the vestibular fibers preserve the ability to respond to sounds [Didier et al., 1989; Mc Cue et al., 1994; Murofushi et al.1995; Wu and Murofushi, 1999; Ochi et al., 2001; Matsuzaki et al., 2002; Yang et al., 2005; Basta et al., 2005].

Didier et al. (1989) recorded click-evoked potentials in guinea pigs with impaired cochlea and unharmed vestibular system. Murofushi et al. (1995) demonstrated that the primary afferent pathway originating from the saccule in guinea-pigs responds to short-length and high-intensity stimulations. Matsuzaki and Murofushi (2002) reported the presence of potentials evoked by clicks, recorded through electrodes put on the neck of anesthetized guinea pigs, without any contraction of the cervical muscles, which indicates that the negative peak originates from neurogenic activity of the vestibular-spinal tract. Yang and Young (2005) recorded myogenic potentials in alert guinea pigs, proving their vestibular origin, and compared the results of two groups, the first with a normal vestibular system, (i.e., VEMPs normally present), and the second after the administration of gentamicin and the disappearance of VEMPs.
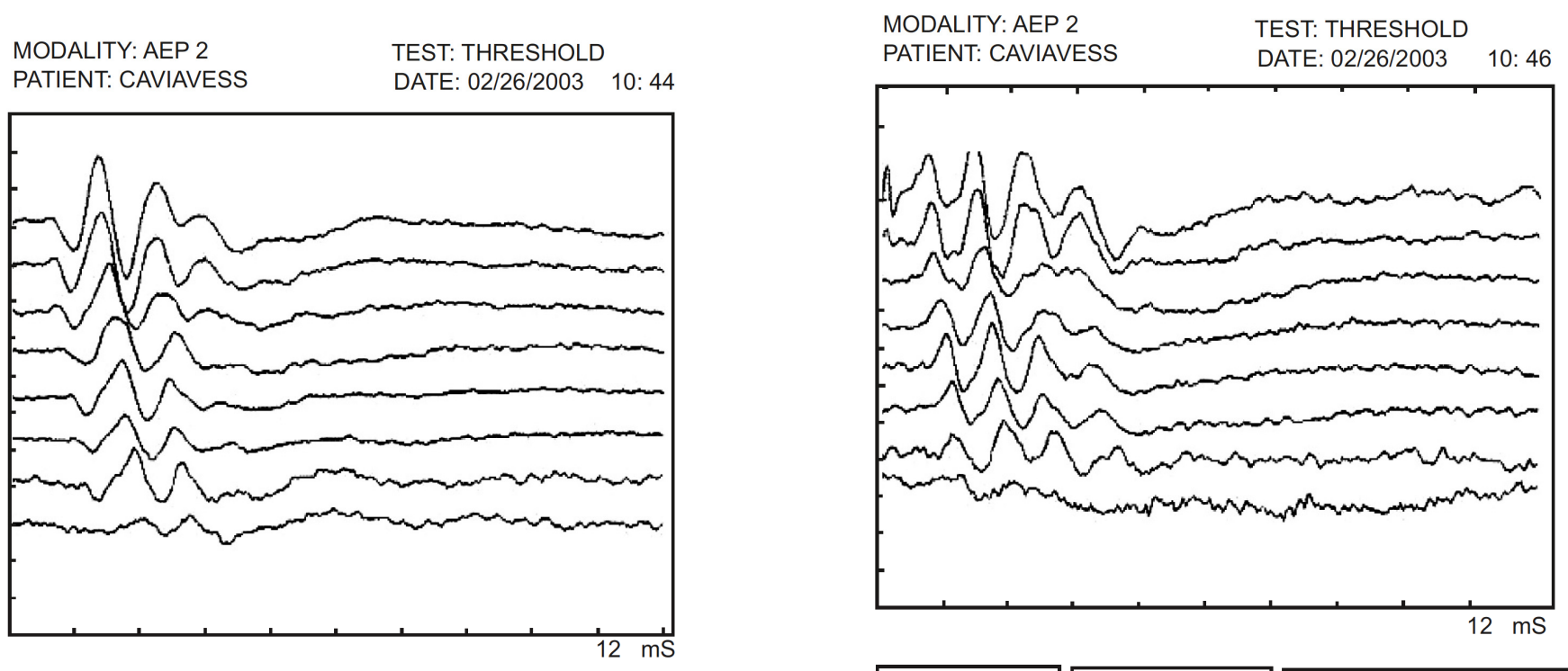

Fig. (1). Normal ABR responses during anesthesia.

Table 1. ABR - Absolute and Inter-Peak Latencies Averaging in the Four Groups of Anesthetized Guinea-Pigs, Divided in Four Groups, According to the Anesthesiologic Protocol Employed

\begin{tabular}{|c|c|c|c|c|c|c|}
\hline Peak Latencies & $\mathbf{I}$ & II & III & IV & I-III & I-IV \\
\hline Group 1 (4 guinea pigs) & $0.876 \pm 0.0046$ & $1.780 \pm 0.0126$ & $2.622 \pm 0.0060$ & $3.514 \pm 0.0122$ & $1.746 \pm 0.0066$ & $2.710 \pm 0.0143$ \\
\hline Group 3 (4 guinea pigs) & $0.852 \pm 0.0028$ & $1.584 \pm 0.0118$ & $2.424 \pm 0.0106$ & $3.360 \pm 0.0118$ & $1.692 \pm 0.0106$ & $2.508 \pm 0.0153$ \\
\hline Group 4 (4 guinea pigs) & $0.840 \pm 0.0024$ & $1.608 \pm 0.0083$ & $2.532 \pm 0.0084$ & $3.408 \pm 0.0104$ & $1.584 \pm 0.0096$ & $2.543 \pm 0.0126$ \\
\hline
\end{tabular}


Table 2. VEMPs - Responses in Guinea Pigs Before and After Anesthesia (there were No Differences in VEMP Recordings Before and After Anesthesia)

\begin{tabular}{|c|c|c|c|}
\hline Peak Latencies & I & II & III \\
\hline \hline Group 1 (4 guinea pigs) & $8.425 \pm 0.263$ & $11.80 \pm 0.282$ & $16.50 \pm 0.259$ \\
\hline Group 2 (4 guinea pigs) & $7.56 \pm 0.234$ & $11.225 \pm 0.303$ & $15.35 \pm 0.191$ \\
\hline Group 3 (4 guinea pigs) & $8.05 \pm 0.208$ & $10.95 \pm 0.191$ & $15.00 \pm 0.230$ \\
\hline Group 4 (4 guinea pigs) & $7.88 \pm 0.258$ & $10.90 \pm 0.258$ & $14.90 \pm 0.206$ \\
\hline
\end{tabular}

MODALITY: AEP

PATIENT: CAVIAVESS
TEST: MIDDLE AEP

DATE: 02/26/2003
10: 59

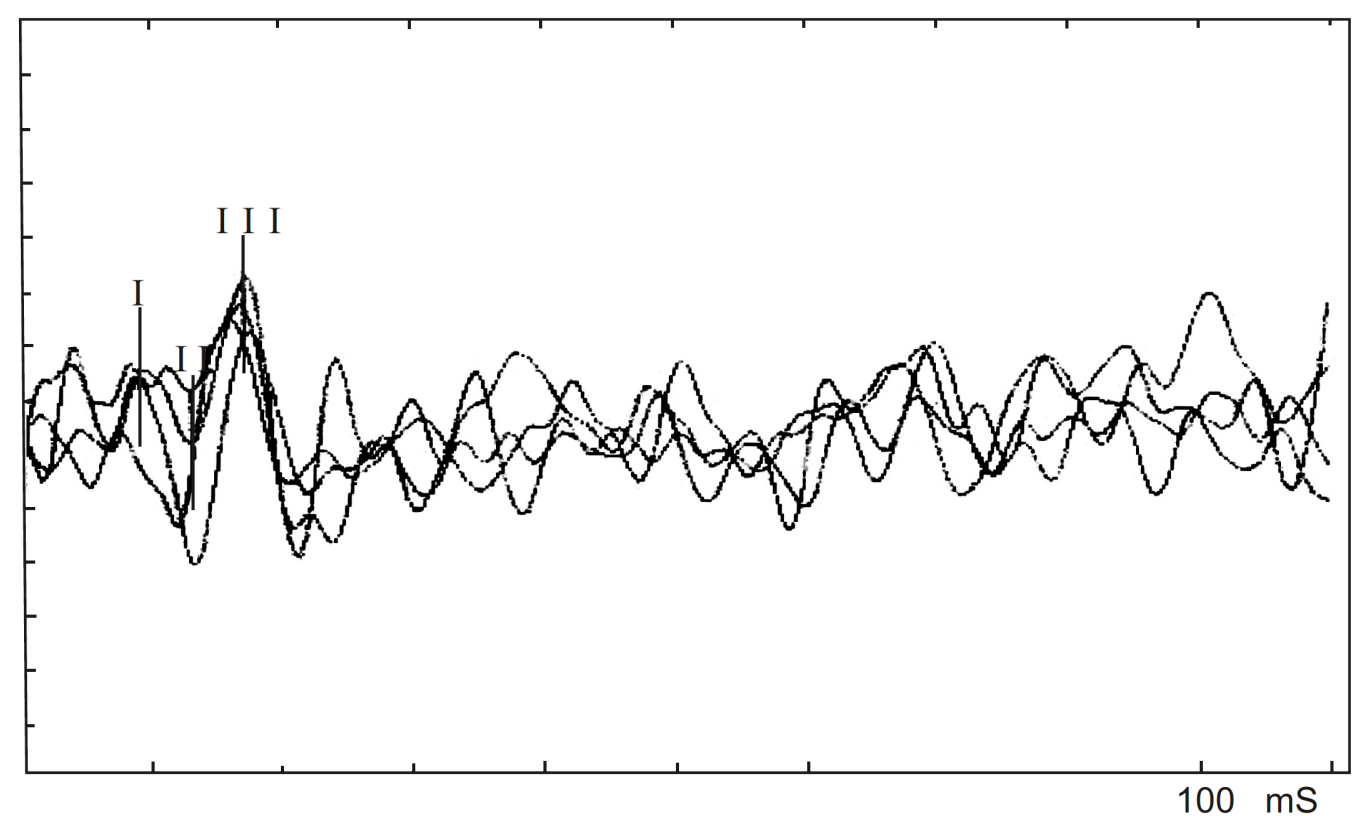

Fig. (2). VEMP responses in guinea pigs before anesthesia and at awakening.
MODALITY: AEP
PATIENT: CAVIAVESS
TEST: MIDDLE AEP
DATE: 02/26/2003

10: 35

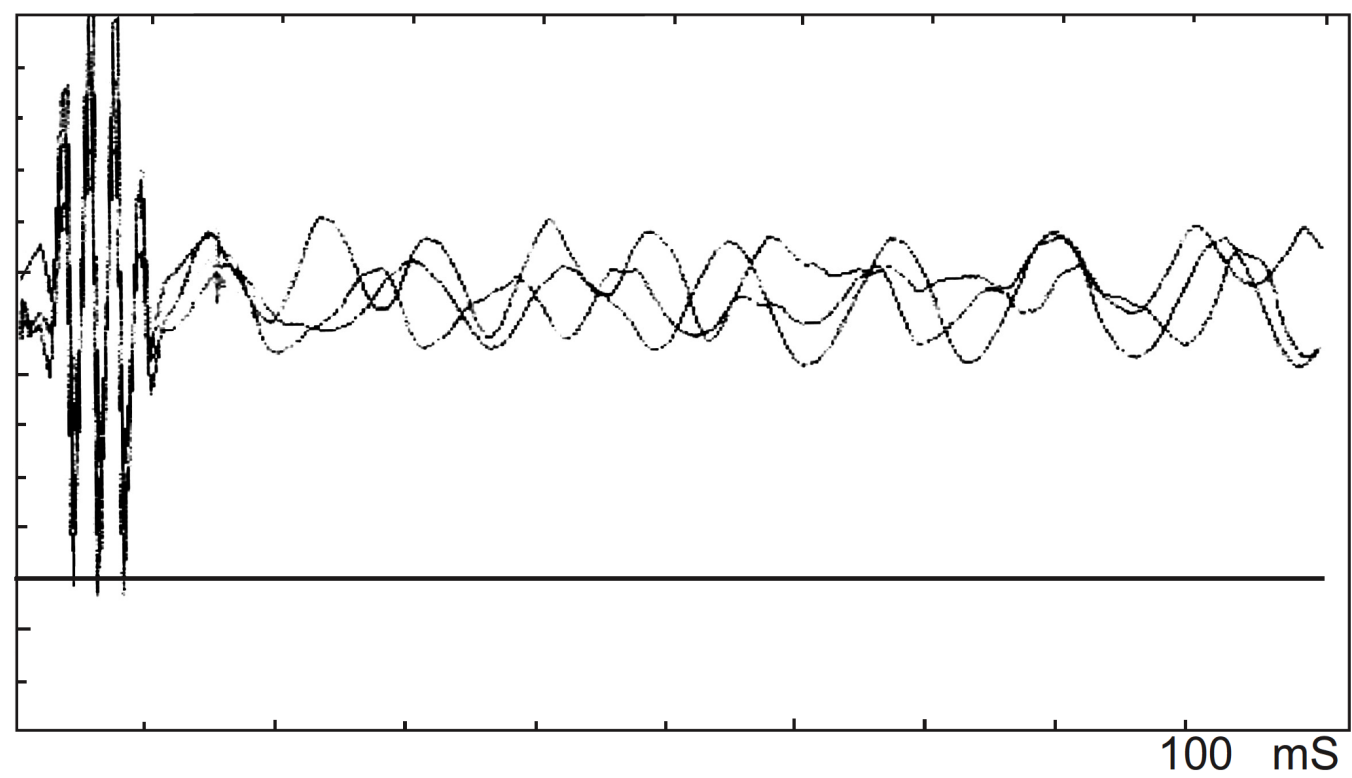

Fig. (3). Total absence of VEMP responses in guinea pigs during anesthesia. 
Table 3. Statistical Correlation of ABR Responses Between Different Groups of Anesthetized Guinea-Pigs

\begin{tabular}{|c|c|c|c|c|c|}
\hline Peak Latencies & I & III & IV & I-III & I-IV \\
\hline \hline groups 1 -2 & 0.020029 & 0.162892 & 0.116920 & 0.036473 & 0.116503 \\
\hline groups 1-3 & 0.114129 & 0.095559 & 0.129114 & 0.021088 & 0.081061 \\
\hline groups 1-4 & 0.195129 & 0.001448 & 0.051661 & 0.016000 & 0.039124 \\
\hline groups 2-3 & 0.066987 & 0.170699 & 0.043376 & 0.038176 & 0.024940 \\
\hline groups 2-4 & 0.012004 & 0.05012 & 0.018925 & 0.091266 & 0.036834 \\
\hline groups 3-4 & 0.168482 & 0.080431 & 0.028138 & & 0.0689 \\
\hline
\end{tabular}

Table 4. Statistical Correlation of VEMP Responses Before and After Anesthesia

\begin{tabular}{|c|c|c|c|}
\hline Peak Latencies & t-Test P1 & t-Test N1 & t-Test P2 \\
\hline \hline group 1 (4 guinea pigs) & 0.055348 & 0.020678 & 0.009561 \\
\hline group 2 (4 guinea pigs) & 0.032141 & 0.098871 & 0.086154 \\
\hline group 3 (4 guinea pigs) & 0.066036 & 0.007505 & 0.004976 \\
\hline group 4 (4 guinea pigs) & 0.016017 & 0.002330 & 0.001226 \\
\hline
\end{tabular}

On the basis of these results, demonstrating the vestibular origin of myogenic potentials, we aimed to evaluate, in an animal model, the effect exerted by anesthesia in general, and by various anesthetic drugs on the VEMPs recording. We compared the latencies averaging in guinea pigs with those of humans, and, though with a limited difference due to a smaller head size, we found substantial correspondence. In our study, the mean latencies in alert guinea pigs were $7.98 \pm 0.238$ and $11.21 \pm 0.258 \mathrm{msec}$; respectively, for the first positive and negative peak. The restrained position with tension of the neck muscles afforded ample vestibular potentials, which disappeared in all cases during general anesthesia. After complete awakening the potentials reappeared, preserving the same pre-anesthesia characteristics (t-test: 0.05 for group 1; 0.032 for group 2; 0.066 for group 3; 0.016 for group 4), but with different awakening times due to the different action mechanisms of the drugs. The statistical analysis performed on the responses at awakening of the four groups showed no statistically significant differences. The ABR in all cases were normal. The comparisons of the four groups did not show significant variations, confirming that different anesthetic drugs do not influence the response parameters. The significance level of our statistical analysis was set for $\mathrm{p}<0,05$.

Our study shows that anesthetic drugs invariably cause the disappearance of saccular reflexes. The emerged similarity between the values for guinea pigs and those available in the literature for humans constitutes the basis and stimuli for future studies on the particular aspects of the sacculocollic reflex, with a possible application in clinical practice.

\section{REFERENCES}

[1] Murofushi T, Halmagyi GM, Yavor RA, Colebatch JG. Absent vestibular evoked myogenic potentials in vestibular neurolabyrinthitis. An indicator of inferior vestibular nerve involvement? Arch Otolaryngol Head Neck Surg 1996; 122: 84548.

[2] Ferber-Viart C, Dubreuil C, Duclaux R. Vestibular evoked myogenic potentials in humans: A Review. Acta Otolaryngol 1999; 119: 6-15.

[3] Wang C, Young YH. Earlier and later components of tone burst evoked myogenic potentials. Hear Res 2004; 191: 59-66.

[4] Wu CC, Young YH. Vestibular evoked myogenic potentials are intact after sudden deafness. Ear Hearing 2002; 23: 235-38.

[5] Yang TH, Young YH. Click-evoked myogenic potentials recorded on alert guinea pigs. Hear Res 2005; 205: 277-83.

[6] Matsuzaki M, Murofushi T. Click-evoked potentials on the neck of the guinea pig. Hear Res 2002; 165: 152-55.

[7] $\mathrm{Wu} \mathrm{CH}$, Murofushi T. The effect of click repetition rate on vestibular evoked myogenic potential. Acta Otolaryngol 1999; 119 (1): 29-32.

[8] Ochi K, Ohashi T, Nishino H. Variance of vestibular evoked myogenic potentials. Laryngoscope 2001; 111: 522-27.

[9] Welgampola MS, Colebatch JG. Characteristics and clinical applications of vestibular evoked myogenic potentials. Neurology 2005; 64: 1682-88.

[10] Murofushi T, Iwasaki S. Recovery of vestibular evoked myogenic potentials. Acta Otolaryngol 2006; 126: 364-7.

[11] Duwel P, Walther LE. Otolith Function Test: A Differentiated, Quality-Assured Screening System. Laryngorhinootologie 2005; 84: 653-9.

[12] Young YH. Vestibular evoked myogenic potentials in delayed endolymphatic hydrops. Laryngoscope 2002; 112: 1623-6.

[13] Lu YC, Young YH. Vertigo from herpes zoster oticus: superior or inferior vestibular origin? Laryngoscope 2003; 113: 307-11.

[14] Morawiec-Bajda A, Wasilewski B. Myogenic vestibular evoked potential used to objective estimation of effectiveness of central action drugs. Otolaryngol Pol 2000; 54(3): 327-3.

[15] Basta D, Todt I, Ernst A. Normative data for p1/n1-latencies of vestibular evoked myogenic potentials induced by air or boneconducted tone bursts. Clin Neurophysiol 2005; 116: 2216-19. 